\title{
Positive- and negative-tone structuring of crystalline silicon by laser-assisted chemical etching
}

\author{
M. Chambonneau, ${ }^{1, *}$ (i) X. Wang, ${ }^{2}$ X. Yu, ${ }^{2,3}$ Q. Li, ${ }^{1}$ (i) D. Chaudanson,,${ }^{4}$ S. Lei, ${ }^{2}$ and D. Grojo ${ }^{1}$ \\ ${ }^{1}$ Aix-Marseille University, CNRS, LP3, F-13288 Marseille, France \\ ${ }^{2}$ Department of Industrial and Manufacturing Systems Engineering, Kansas State University, Manhattan, Kansas 66502, USA \\ ${ }^{3}$ CREOL, The College of Optics and Photonics, University of Central Florida, Orlando, Florida 32816, USA \\ ${ }^{4}$ Aix-Marseille University, CNRS, CINaM UMR 7325, F-13288 Marseille, France \\ ${ }^{*}$ Corresponding author: maxime.chambonneau@lp3.univ-mrs.fr
}

Received 26 December 2018; revised 21 February 2019; accepted 24 February 2019; posted 25 February 2019 (Doc. ID 356162); published 21 March 2019

\begin{abstract}
We demonstrate a structuring method for crystalline silicon using nanosecond laser internal irradiation followed by chemical etching. We show a dramatic dependence of the etch rate on the laser-writing speed. Enhanced isotropic etch rates of silicon by laser-induced internal damage were recently demonstrated with strong acids, but our results add the possibility to obtain reduced etch rates leading to different topographies. Material analyses indicate the possibility to efficiently produce high-aspect ratio channels, thanks to laser-induced porosities, as well as silicon microbumps due to highly stressed regions. This holds promises for fabricating microfluidic, photovoltaic, and microelectromechanical systems. (2019 Optical Society of America
\end{abstract}

https://doi.org/10.1364/OL.44.001619

Provided under the terms of the OSA Open Access Publishing Agreement

Nonlinear processes (especially the plasma defocusing phenomenon) impose severe limits on the laser energy that can be deposited inside crystalline silicon (c-Si) with femtosecond pulses [1], and make material modification with individual pulses extremely challenging [2]. The advent of high-power nanosecond infrared (IR) laser sources has helped to circumvent these limitations [3,4]. Advanced characterizations of the laser-modified materials with a constant focus have shed light on a wide variety of crystalline structures induced in the bulk of $\mathrm{c}-\mathrm{Si}$ by these pulses, including polycrystalline phases [5], voids [6], and high-pressure phases [7]. This diversity is similar to what can be produced on the surface of silicon at wavelengths below $1.1 \mu \mathrm{m}$ for which the material is not transparent [8-10]. It also has been established that the internal modifications are accompanied by a change of the apparent refractive index of silicon. Accordingly, various optical functionalities have been directly conferred by laser writing to bulk c-Si [11-13]. However, efforts are still required for establishing the relationship between the structural changes and the index modifications. Moreover, the knowledge and control of the material structural aspects would open a path to the fabrication of three-dimensional (3D) architectures in c-Si by combining in-volume laser writing and chemical etching, just as was done for fused silica after the first waveguides were fabricated [14-16]. In comparison to the previously reported laser-assisted chemical etching methods on the surface of silicon $[17,18]$, such a technique would also add the interesting benefits of the absence of surface plasma effect (e.g., shielding) and/or debris. Making a first step in this direction, enhanced etch rates for strong acid solutions have been reported recently in the nanosecond laser damage regime, helping in the penetration of the etchant deep inside the material [12]. This allowed the authors to demonstrate negative-tone fabrication (i.e., the laserirradiated zone is removed) of complex 3D structures, including microfluidic channels, vias, and micro-electromechanical systems [12].

Supported by advanced material analyses, we demonstrate in this Letter an extra level of flexibility by adding the possibility for positive-tone microfabrication (i.e., the laser-irradiated zone remains after development) with this general approach. While we have investigated the influence of several laser parameters, we show how the laser-writing speed, an easily adjustable parameter, can be exploited to fabricate not only high-aspect ratio negative structures, but also bumps after a subsequent etching step in a diluted potassium hydroxide $(\mathrm{KOH})$ solution. This evidences that not only the magnitude, but also the sign of the $\mathrm{c}-\mathrm{Si}$ etch rate change in a standard $\mathrm{KOH}$ solution can be tailored with this control parameter. Using electron diagnostics and micro-Raman spectroscopy, the difference is explained by the production of material disruptions in the first case and stressed monocrystalline zones inside the material in the second one. These two types of material modifications simply help or prevent the penetration of the chemical solution deep inside the material. Interestingly, the conditions to create the densified zones are the exact analog to those used for the fabrication of the first waveguides by positive refractive index engineering in c-Si [11]. This flexible laser-based approach for positive and negative texturing of c-Si surfaces may find plenty of applications in microfluidics, including the preparation of 
superhydrophobic substrates [19], channel-based microfluidic devices, black silicon for photovoltaics application [20], as well as the fabrication of lab-on-a-chip systems [16].

The experimental setup for inscribing modifications inside $\mathrm{c}-\mathrm{Si}$ is detailed in Ref. [11]. Described briefly, this arrangement relies on pulses at $1.55 \mu \mathrm{m}$ wavelength emitted by an Er-doped laser source operated at a $1 \mathrm{kHz}$ repetition rate. The temporal profile is Gaussian with a pulse duration of $5 \mathrm{~ns}$ (full width at half-maximum). The laser energy is adjusted by combining a half-wave plate and a polarizer. The beam is focused inside a $1 \mathrm{~mm}$ thick intrinsic c-Si sample [(100)-oriented] with an objective lens of numerical aperture $\mathrm{NA}=0.42$ mounted on motorized stages. The diameter at $1 / e^{2}$ of the Gaussian beam is $\sim 2.3 \mu \mathrm{m}$ at the focus, and the Rayleigh length in air is $\sim 2.8 \mu \mathrm{m}$. The focal spot is uniformly moved longitudinally from the rear to the front surface at $v=1.0$ and $0.1 \mathrm{~mm} / \mathrm{s}$, corresponding to 5.6 and 56 pulses per point of the material, respectively, according to the focusing geometry. As shown in Ref. [11], the morphology of the lines produced at $1.0 \mathrm{~mm} / \mathrm{s}$ mainly consists of voids (with a negative refractive index change), whereas the lines inscribed at $0.1 \mathrm{~mm} / \mathrm{s}$ exhibit a homogeneous positive refractive index. For writing speeds in between and lower than $0.1 \mathrm{~mm} / \mathrm{s}$, a combination of these two morphologies likely provoked by cumulative effects was observed [11]. Therefore, the speeds of 1.0 and $0.1 \mathrm{~mm} / \mathrm{s}$ have been selected in this Letter in order to study two single types of refractive index change (i.e., positive and negative). Although damage is systematically observed on the front surface, no modification is detected on the rear surface for all the pulse energies used between 2.7 and $0.5 \mu \mathrm{J}$ (measured at the focus in air prior to the experiments). This range was chosen in order to produce modifications inside silicon with the maximum reachable energy with our setup down to an energy close to the bulk damage threshold $[4,11]$. For ensuring repeatability, six lines have been inscribed using identical processing conditions. It must be emphasized that, as shown in Ref. [11], the general morphology of the written lines does not change with the pulse energy, except for the line diameter. After laser writing inside $\mathrm{c}-\mathrm{Si}$, the modifications are observed by means of a customized phase-shift interferometry microscope described in Ref. [21]. Briefly, this microscope relies on an IR MachZehnder interferometer and provides quantitative information about the refractive index change of the modifications. The sample is then polished on the exit surface and treated by anisotropic chemical etching during $90 \mathrm{~min}$ using a $22 \mathrm{wt}$. \% $\mathrm{KOH}$ solution at the temperature of $40^{\circ} \mathrm{C}$, mixed with a saturated $20 \mathrm{wt}$. \% isopropyl alcohol solution. The corresponding $\langle 100\rangle$ etch rate measured experimentally on a silicon sample unmodified by the laser is $10 \mu \mathrm{m} / \mathrm{h}$. The rear surface is then characterized in Nomarski interference contrast (NIC) microscopy, scanning electron microscopy (SEM), transmission electron microscopy (TEM) and electron diffraction after a lamella preparation using a focused ion beam (FIB), as well as Raman spectroscopy.

The typical morphology of the lines written in the bulk of c-Si is shown in Figs. 1(a) and 1(b) for two different writing speeds. These images are acquired before chemical etching by means of the IR phase-shift microscope. At a writing speed of $v=1.0 \mathrm{~mm} / \mathrm{s}$, the line is likely composed of randomly distributed voids as emphasized by the dark spots in (a) corresponding to a negative phase shift. Conversely, for $v=0.1 \mathrm{~mm} / \mathrm{s}$, the

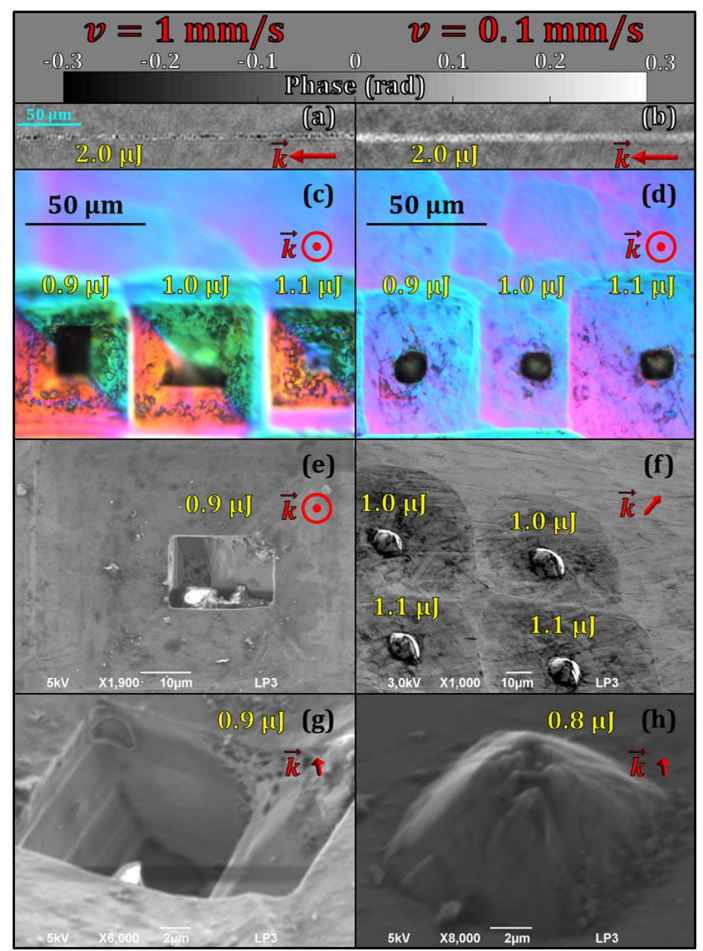

Fig. 1. (a), (b) Typical IR phase micrographs of modifications in bulk silicon written at 1.0 and $0.1 \mathrm{~mm} / \mathrm{s}$, respectively. These are obtained before etching, and the scale bar applies to both images. (c), (d) are the NIC micrographs of the surface after etching. (e), (f), (g), and (h) are SEM characterizations of this surface. The vector $\vec{k}$ shows the laser direction of propagation.

line in (b) consists of a homogeneous positive phase shift, as confirmed by the waveguiding properties of the structures written under the same conditions [11]. In this case, one may note a slight negative phase shift of about -0.05 rad surrounding the positive one. This morphological difference in the bulk is also revealed on the surface after $\mathrm{KOH}$ etching, as shown by the NIC micrographs in (c) and (d). Indeed, the etching of the modification written at $1.0 \mathrm{~mm} / \mathrm{s}$ results in an inverted pyramid, including a hole in its center [see the dark rectangles in (c)]. Thanks to the NIC technique, a strong color contrast is exhibited in the inverted pyramid area in comparison with the non-irradiated material on top of the image suggesting an important change of the surface slope. This morphology is observed for all the tested energies, showing that it only depends on the laser-writing speed. Nevertheless, one can note that, as suggested in (c), the size and depth of the holes do not depend on laser energy. This may be attributed to the irregular spatial distribution of the voids along the optical axis, as shown in (a) which is found to be a typical feature at this writing speed and independent of the laser energy. This surface morphology is confirmed by the SEM images in (e) and (g), exhibiting an inverted pyramid with the four well-defined edges, as well as the deep hole in the center. This is a typical feature of $\mathrm{KOH}$, a common etchant for $\mathrm{Si}$ and associated with a reaction rate on the $\langle 100\rangle$ planes much higher than on the $\langle 111\rangle$ planes. This leads to this anisotropic etching initiated by any local window for the etchant and already widely exploited for surface engineering for various applications [22]. 
In contrast, surface morphology around the modification written at $0.1 \mathrm{~mm} / \mathrm{s}$, is extremely different, as highlighted by the NIC micrograph in (d). First, the etching depth around the modification is much smaller than in (c), since the aforementioned color contrast is similar to that of the non-irradiated area on top of the image. Secondly, the central area is more repeatable and regular than in (c). Interestingly, the SEM images in (f) and (h) show that the central area corresponding to the positive phase shift in (b) is a bump exhibiting also a pyramidal shape somehow opposite to the previously developed hole morphology associated with the lines written at $1.0 \mathrm{~mm} / \mathrm{s}$.

The difference in surface morphology between the lines written at the two different speeds can be attributed to the penetration ability of the $\mathrm{KOH}$ solution inside the laser-modified material. Indeed, the observed positive phase shift (and, thus, the positive refractive index change $\Delta n$ ) in Fig. 1(b) could result from a local densification provoked by laser-induced stress inside the material. This would imply that the penetration of the chemical solution is slow within the zone where $\Delta n>0$, in agreement with the SEM observations in (f) and (h). The pyramidal morphology in Fig. 1(h) first suggests that the stress is more important in the center of the line with $\Delta n>0$ than in its periphery since if it was homogeneously distributed, a cylinder would be exhibited in (h). Second, this tetrahedral shape rather than a conical one implies that the material mainly remains monocrystalline; so the anisotropy of $\mathrm{KOH}$ etching keeps following the crystalline axes of the silicon sample. Ultimately, the $20 \mu \mathrm{m}$ radius area with a slight negative phase shift surrounding the line where $\Delta n>0$ indicates that the material has been rarefied in this zone. This is very consistent with the slightly ablated square around the pyramid in comparison with the non-laser-processed surface in (d) and (f). Conversely, for the highest speed $(v=1.0 \mathrm{~mm} / \mathrm{s})$ voids are produced [see Fig. 1(a)] and can help the chemical solution to penetrate from the surface deep inside the material. This is consistent with the holes observed in (c), (e), and (g).

In order to validate this scenario, high resolution characterizations of the two aforementioned morphologies are performed using electron microscopy, providing paramount information about the nature of the laser-produced modifications. To do so, a cross section of a hole similar to these displayed in Figs. 1(c) and 1(e) is prepared thanks to ion polishing. The resulting SEM images are shown in Fig. 2, where a secondary electrons image in (a) is compared to a backscattering electron image in (b). The main difference between these two imaging

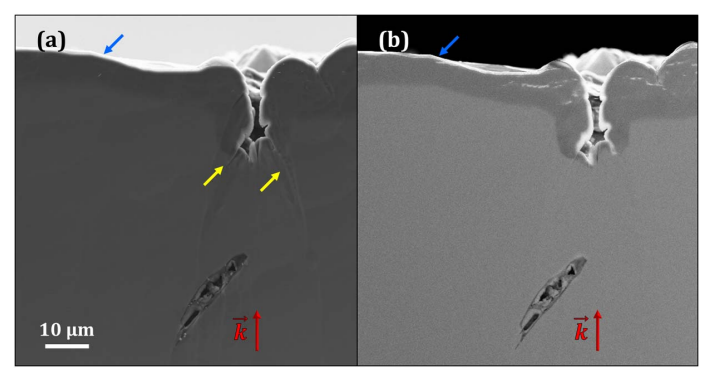

Fig. 2. SEM image of an etched site written at $1 \mathrm{~mm} / \mathrm{s}$ and $0.9 \mu \mathrm{J}$ obtained with (a) secondary electrons and (b) backscattering electrons. The vector $k$ shows the laser direction of propagation. The blue and yellow arrows indicate the sample surface and subsurface cracks, respectively. The spatial scale applies to both images. techniques is that (a) is sensitive to the surface topography while (b) is sensitive to the atomic mass of the elements. Both of these images reveal the high-aspect ratio of the hole which is $\sim 24 \mu \mathrm{m}$ deep under the surface unmodified by the laser for a minimum diameter of $\sim 3 \mu \mathrm{m}$. The measured depth of the hole enables us to estimate the etch rate in the laser-modified area. This etch rate is obtained by measuring the height difference between (i) the surface before and after etching, and (ii) the surface after etching and the bottom of the hole in Fig. 2. This etch rate is $\sim 22.7 \mu \mathrm{m} / \mathrm{h}$, i.e., more than $\sim 2.3$ higher than for unmodified silicon. Moreover, one can observe a void structure in accordance with Ref. [6] at about $40 \mu \mathrm{m}$ below the surface consistent with our conclusions drawn from the phase microscopy in Fig. 1(a). At the bottom of the hole, one can distinguish cracks in Fig. 2(a) which likely have been assisting the $\mathrm{KOH}$ solution to penetrate inside the material. Interestingly, Fig. 2(b) reveals a dark grey layer of $\sim 7 \mu \mathrm{m}$ starting from the surface, suggesting a material modification at the surface layer. We attribute this feature to the exothermic character of the etching process.

Ultimately, electron diagnostics are performed on the pyramidal morphology exhibited in Figs. 1(f) and 1(h). The corresponding results are shown in Fig. 3. A TEM lamella from the central portion of the modified site as indicated by the red dotted line in (a) is prepared using FIB. Analogous to the observations in Fig. 2, the lamella allows us to evaluate the height of the pyramid of $\sim 5.1 \mu \mathrm{m}$ with respect to the surface unmodified by the laser. This corresponds to an etch rate of $\sim 6.6 \mu \mathrm{m} / \mathrm{h}$. The TEM characterization in (b) and the magnified image in (c) clearly reveal the laser-affected zone. Although the modifications look homogeneous at the microscale level

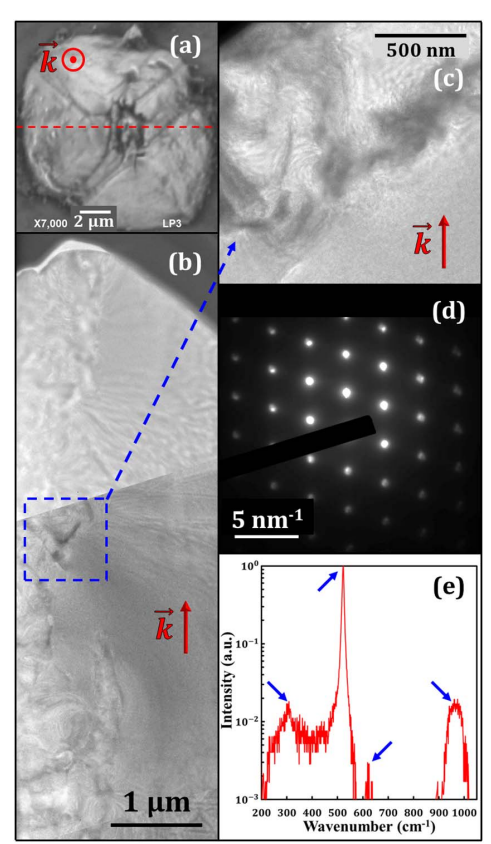

Fig. 3. (a) SEM image of an etched site written at $0.1 \mathrm{~mm} / \mathrm{s}$ and $0.9 \mu \mathrm{J}$. (b) TEM image of the same site corresponding to the dotted red line in (a). (c) TEM magnification of the dotted blue square in (b). The vector $\vec{k}$ shows the laser direction of propagation. (d) Electron diffraction pattern observed in (c). (e) Micro-Raman spectrum of the site observed in (a), with monocrystalline features of silicon according to Ref. [23] indicated by the blue arrows. 
under IR phase-shift microscopy [Fig. 1(b)], the TEM images show irregularly distributed features at the nanoscale along the optical axis. While in Ref. [11] these irregularities were useful for investigating the light-guiding properties of the lines written at $0.1 \mathrm{~mm} / \mathrm{s}$ with scattering, they represent a serious drawback for refractive index engineering in silicon where low losses are required. The observations in (b) and (c) are possible if either (1) the allotropic form of silicon has been modified or (2) stress has been induced inside the material. In order to investigate these hypotheses, electron diffraction measurements are performed. As shown in (d), the diffraction pattern is typical of monocrystalline silicon. Moreover, this pattern repeated at several points is the same as that from the (100)-oriented c-Si far from the laser-affected zone. This is all the more supported by micro-Raman measurements shown in (e) revealing the typical features of monocrystalline silicon indicated by the arrows. These Raman signatures of monocrystalline silicon are (1) the main peak at $520 \mathrm{~cm}^{-1}$, (2) the secondary peaks at 300 and $610 \mathrm{~cm}^{-1}$, and (3) a hill from about $910-1100 \mathrm{~cm}^{-1}$ [23]. The Raman spectrum is the same for the pyramidal area and the unmodified silicon. It is worth noting in (e) the absence of a broad band around $480 \mathrm{~cm}^{-1}$ inherent to amorphous silicon [24]. Therefore, we conclude from (d) and (e) that the nanosecond-laser irradiation does not affect the crystalline structure of c-Si. Nevertheless, based on the TEM images of the laser-modified zone in Figs. 3(b) and 3(c), as well as the positive refractive index change measured in Fig. 1(b), the modified region is likely to contain highly stressed materials. Indeed, such a stressed zone in the bulk can be provoked by shock waves induced by the pulses [25]. No void is visible under phase microscopy in this case [see Fig. 1(b)] with the writing speed of $0.1 \mathrm{~mm} / \mathrm{s}$ which, according to our focusing conditions, implies that the motion of the melted material left by one pulse fills the voids left by the preceding ones [11]. Thereby, the results in Fig. 3 not only provide information about the crystalline structure of the lines written at $0.1 \mathrm{~mm} / \mathrm{s}$ with a positive refractive index change, but also imply that the waveguide properties are induced by stressed material following the laser path, similar to observations in fused silica [26]. Finally, in contrast to the voids left at $1.0 \mathrm{~mm} / \mathrm{s}$, the strained material at $0.1 \mathrm{~mm} / \mathrm{s}$ limits the penetration of the $\mathrm{KOH}$ solution, resulting in a bump morphology, as shown in Fig. 1.

To summarize, a surface texturing method for c-Si has been introduced. This technique relies on in-volume structuring with nanosecond laser pulses, followed by chemical etching of the surface with $\mathrm{KOH}$. By only adjusting the speed of the laser-writing process, positive and negative texturing can be produced on the surface after chemical etching. In a more fundamental way, material characterizations show that the lines written at a high speed consist of voids facilitating the penetration of the $\mathrm{KOH}$ solution deep inside silicon. On the other hand, the lines written at a moderate speed remain unchanged in terms of crystalline structure, but become densified due to laser-induced stress. Interestingly, this indirectly reveals the material science aspect behind the waveguide properties recently reported for these structures. By limiting the penetration of etchants in comparison to the zone non-affected by the laser, the same regime offers today a new possibility for positive-tone microfabrication making laser-assisted chemical etching a more flexible method for silicon applications. To go further in the comprehension, the same process will be applied for various crystal orientations at different writing speeds in the future for studying the morphology of the modified zone after both laser writing and chemical etching.

Funding. H2020 European Research Council (ERC) (724480); National Science Foundation (NSF) (CMMI1537846).

\section{REFERENCES}

1. E. V. Zavedeev, V. V. Kononenko, and V. I. Konov, Laser Phys. 26 016101 (2015).

2. M. Chanal, V. Yu. Fedorov, M. Chambonneau, R. Clady, S. Tzortzakis, and D. Grojo, Nat. Commun. 8, 773 (2017).

3. E. Ohmura, F. Fukuyo, K. Fukumitsu, and H. Morita, J. Achiev. Mater. Manuf. Eng. 17, 381 (2006).

4. P. C. Verburg, G. R. B. E. Römer, and A. J. Huis in 't Veld, Opt. Express 22, 21958 (2014).

5. P. C. Verburg, L. A. Smillies, G. R. B. E. Römer, B. Haberl, J. E. Bradby, J. S. Williams, and A. J. Huis in 't Veld, Appl. Phys. A 120, 683 (2015).

6. H. Iwata, D. Kawaguchi, and H. Saka, Microscopy 66, 328 (2017).

7. H. Iwata, D. Kawaguchi, and H. Saka, Microscopy 67, 30 (2018).

8. T. H. R. Crawford, J. Yamanaka, G. A. Botton, and H. K. Haugen, J. Appl. Phys. 103, 053104 (2008).

9. L. Rapp, B. Haberl, C. J. Pickard, J. E. Bradby, E. G. Gamaly, J. S. Williams, and A. V. Rode, Nat. Commun. 6, 7555 (2015).

10. J. Mur, L. Pirker, N. Osterman, and R. Petkovšek, Opt. Express 25, 26356 (2017).

11. M. Chambonneau, Q. Li, M. Chanal, N. Sanner, and D. Grojo, Opt. Lett. 41, 4875 (2016).

12. O. Tokel, A. Turnalı, G. Makey, P. Elahi, T. Çolakoğlu, E. Ergeçen, Ö. Yavuz, R. Hübner, M. Zolfaghari Borra, I. Pavlov, A. Bek, R. Turan, D. K. Kesim, S. Tozburun, S. Ilday, and F. Ö. Ilday, Nat. Photonics 11, 639 (2017).

13. M. Chambonneau, D. Richter, S. Nolte, and D. Grojo, Opt. Lett. 43 6069 (2018)

14. A. Marcinkevičius, S. Juodkazis, M. Watanabe, M. Miwa, S. Matsuo, H. Misawa, and J. Nishii, Opt. Lett. 26, 277 (2001).

15. R. S. Taylor, C. Hnatovsky, E. Simova, D. M. Rayner, V. R. Bhardwaj, and P. B. Corkum, Opt. Lett. 28, 1043 (2003).

16. Y. Bellouard, A. Said, M. Dugan, and P. Bado, Opt. Express 12, 2120 (2004).

17. K. W. Kolasinski, D. Mills, and M. Nahidi, J. Vac. Sci. Technol. A 24 , 1474 (2006).

18. M. Saito and S. Kimura, AIP Adv. 7, 025018 (2017).

19. T. Baldacchini, J. E. Carey, M. Zhou, and E. Mazur, Langmuir 22 4917 (2006)

20. J. Oh, H.-C. Yuan, and H. M. Branz, Nat. Nanotechnol. 7, 743 (2012).

21. Q. Li, M. Chambonneau, M. Chanal, and D. Grojo, Appl. Opt. 55, 9577 (2016).

22. A. Kiani, K. Venkatakrishnan, B. Tan, and V. Venkataramanan, Opt. Express 19, 10834 (2011).

23. P. A. Temple and C. E. Hathaway, Phys. Rev. B 7, 3685 (1973).

24. J. Bonse, K.-W. Brzezinka, and A. J. Meixner, Appl. Surf. Sci. 221, 215 (2003).

25. Y. B. Zel'dovich and Y. P. Raizer, Physics of Shock Waves and HighTemperature Hydrodynamic Phenomena (Academic, 1966).

26. V. R. Bhardwaj, P. B. Corkum, D. M. Rayner, C. Hnatovsky, E. Simova, and R. S. Taylor, Opt. Lett. 29, 1312 (2004). 